University of Wollongong

Research Online

Faculty of Informatics - Papers (Archive)

Faculty of Engineering and Information

Sciences

$1-6-2006$

\title{
An impulse ultra-wideband system capable of concurrent transmission and reception, part II: design and performance
}

\author{
Xiaojing Huang \\ University of Wollongong, huang@uow.edu.au \\ Darryn Lowe \\ University of Wollongong, darrynl@uow.edu.au \\ Ricardo Gandia \\ University of Wollongong \\ Eryk Dutkiewicz \\ University of Wollongong, eryk@uow.edu.au
}

Follow this and additional works at: https://ro.uow.edu.au/infopapers

Part of the Physical Sciences and Mathematics Commons

\section{Recommended Citation}

Huang, Xiaojing; Lowe, Darryn; Gandia, Ricardo; and Dutkiewicz, Eryk: An impulse ultra-wideband system capable of concurrent transmission and reception, part II: design and performance 2006.

https://ro.uow.edu.au/infopapers/473

Research Online is the open access institutional repository for the University of Wollongong. For further information contact the UOW Library: research-pubs@uow.edu.au 


\title{
An impulse ultra-wideband system capable of concurrent transmission and reception, part II: design and performance
}

\author{
Abstract \\ This second part of the paper presents the system design and performance simulation results of a novel \\ ultra-wideband (UWB) system developed for low power, long range, mid to high data rate mesh \\ networking applications. Firstly, the transceiver architecture is described, and the system parameters are \\ selected and justified. Then, the physical layer (PHY) protocol is introduced briefly. Finally, the simulation \\ results regarding the system performance with different data rates in Gaussian and multipath channels \\ are given. It is concluded that the designed transceiver architecture and PHY protocol are adequate and \\ the developed receiver algorithms are feasible for practical implementation.

\section{Disciplines} \\ Physical Sciences and Mathematics

\section{Publication Details} \\ This paper was originally published as: Huang, X, Lowe, D, Gandia, R \& Dutkiewicz, E, An impulse ultra- \\ wideband system capable of concurrent transmission and reception, part II: design and performance, \\ Proceedings of the 2006 International Conference on Communications, Circuits and Systems, Guilin, \\ Guangzi, China, June 2006, 2, 1256-1260. Copyright 2006 IEEE.
}




\title{
An Impulse Ultra-wideband System Capable of Concurrent Transmission and Reception, Part II: Design and Performance
}

\author{
Xiaojing Huang, Darryn Lowe, Ricardo Gandia and Eryk Dutkiewicz \\ University of Wollongong \\ Northfields Avenue, Wollongong, NSW 2522, Australia
}

\begin{abstract}
This second part of the paper presents the system design and performance simulation results of a novel ultrawideband (UWB) system developed for low power, long range, mid to high data rate mesh networking applications. Firstly, the transceiver architecture is described, and the system parameters are selected and justified. Then, the physical layer (PHY) protocol is introduced briefly. Finally, the simulation results regarding the system performance with different data rates in Gaussian and multipath channels are given. It is concluded that the designed transceiver architecture and PHY protocol are adequate and the developed receiver algorithms are feasible for practical implementation.
\end{abstract}

\section{INTRODUCTION}

Complementary to the standard activities in defining ultrawideband (UWB) technology for high rate short range (IEEE 802.15.3a) as well as low rate longer range (IEEE 802.15.4a) wireless personal area network (WPAN) applications, this paper focuses on a mid to high date rate long range UWB mesh network capable of supporting voice, video and data communications for military and public safety applications. As this targets UWB mesh networking applications in hostile environments, there are a lot of challenges in designing a suitable physical layer (PHY), including high-speed data transmission over severe multipath channels, coexistence with legacy systems and intentional jammers, throughput loss over multiple hops, and low power long range operation.

To overcome these challenges, innovative techniques are incorporated in the new UWB system [1]. Firstly, we combine the complementary code division multiplexing (CCDM) modulation and the multicode interleaved direct sequence (MCIDS) spreading techniques to generate a unique UWB signal. The CCDM makes the signal amplitude Gaussian distributed, just like a natural perturbing noise, and enables scalable data rates. The MCIDS, in conjunction with impulse signaling, provides the additional processing gains needed to achieve low power and long range operation. It also resolves intersymbol interference (ISI) in server multipath channels and facilitates easy and robust channel estimation through the use of pilot signals in the MCIDS blocks. Secondly, with the concurrent transmission and reception $(\mathrm{Tx} / \mathrm{Rx})$, this $\mathrm{PHY}$ enables a revolutionary medium access control (MAC) mechanism for high-throughput mesh networking. Thirdly,

The authors acknowledge support of Motorola Inc. for funding the research described in this paper. despite being based on the impulse radio concept, the new UWB transceiver uses a conventional radio frequency (RF) design that makes the implementation very simple by avoiding the need for impulse correlation at the receiver.

The above innovations differentiate this UWB PHY from the other UWB PHY protocols currently under development for the IEEE 802.15 WPAN standards [2,3]. The coding, modulation and spreading techniques used, and the data rate and range supported by the new UWB PHY make it an alternative wireless local area network (WLAN) solution that, by offering low power and covert operation capability, creates new markets in the military and public safety sectors.

This second part of the paper proposes the transceiver architecture for the novel UWB system, introduces its physical layer protocol, and provides the performance simulation results under different data rates, channel conditions, and modes of operation. In the transceiver architecture and baseband protocol design, efforts are made to achieve the following objectives. The processing gain must be sufficient to combat intentional jamming and ensure low power operation. The data rates must be scalable to suit different services and different channel conditions. The receiver must be able to capture as much as possible the transmitted signal energy to increase the operation range at limited transmitter power. The PHY format must facilitate efficient channel estimation to combat severe channel fading and improve the robustness against mutipath interference, and also have low overhead for efficient pipelined multiple hop packet transmission.

In the following sections, we first describe the transceiver architecture and justify the selection of the system parameters in Section II. A brief description of the physical layer protocol is given in Section III. Section IV reports the simulation results for system performance evaluation. Finally, the conclusions are drawn in Section $\mathrm{V}$ with remarks on the future work we plan to undertake.

\section{TRANSCEIVER ARCHITECTURE}

\section{A. Transceiver block diagram}

For data transmission, the UWB baseband signal is generated in two steps. The first step is to generate an asymptotically Gaussian-distributed data sequence using the CCDM modulation. The second step is to further spread the signal using the MCIDS spreading, making the final spread signal suitable for transmission through a multipath channel. An 
additional spreading ratio will be provided in the second step. After the CCDM modulation and the MCIDS spreading, the spread spectrum signal will go through a pulse shaping circuitry to satisfy a defined transmit mask. Since the pulse width is chosen to be shorter than the chip time, a further processing gain will be provided, as the ultimate bandwidth of the transmitted signal will be determined by the UWB impulse. Finally, the UWB baseband signal is up-converted into a specified frequency band and transmitted by a broad bandwidth antenna. For data reception, the received UWB RF signal is first down-converted into UWB baseband. Then, the UWB baseband signal is passed through a matched-filter (or pulse correlator) to improve the signal-to-noise ratio (SNR). After the MCIDS despreading and the CCDM demodulation, the transmitted data symbols will be received and forwarded to the MAC layer for further processing. When permitted by channel conditions, transmission and reception can proceed concurrently, which enables a pipelined data transmission through multiple hops in the mesh network.

Figure 1 illustrates how to implement this UWB system, which is partitioned into three parts: RF front-end, high-speed baseband, and low-speed baseband. A conventional I/Q RF architecture is used, which simplifies the impulse generation and correlation, and allows easy frequency band allocation. The high-speed baseband includes the MCIDS and the signal synchronization. For a given frequency band, signal bandwidth and maximum channel delay, the system parameters such as carrier frequency, UWB impulse width, chip time, data block size, spreading sequence length and cyclic prefix length are all predefined. Consequently, the high-speed baseband can be implemented together with UWB RF front-end by hardware (such as an ASIC or FPGA). The low-speed baseband includes diversity combining, CCDM, and other baseband signal processing such as data scrambling and CRC checking, which can be implemented in software via a digital signal processor (DSP) or FPGA, such that the overall spreading ratio or data rate is software-definable. This implementation configuration provides the UWB system with an adaptive processing gain or variable data rate capability, making it suitable for the military application in extreme channel conditions. The baseband and transmitted RF signal waveforms are also illustrated in Figure 1 to show that the proposed UWB transceiver is characterized as an impulse radio with carrier.

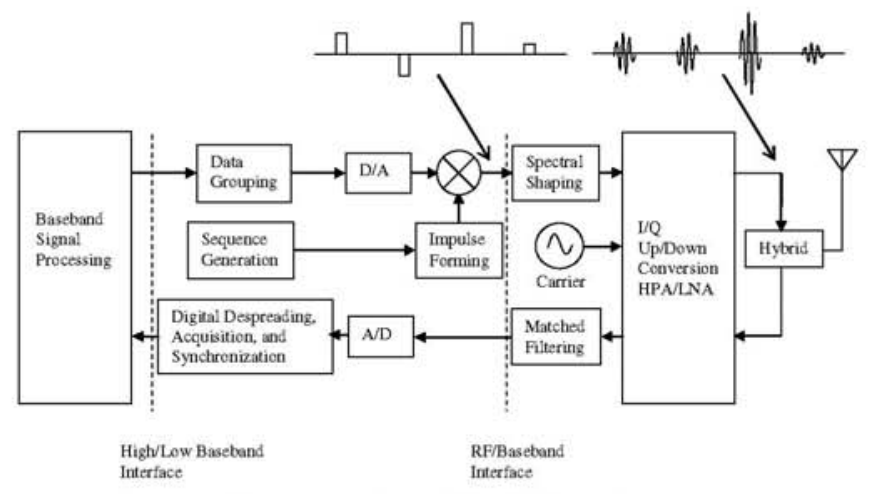

Figure 1. Illustration of new UWB PHY implementation.

\section{B. System parameter selection consideration}

The transmit bandwidth of the new UWB system can be selected depending on application; a high bandwidth can offer higher data rates and/or higher processing gains, but does so at the cost of implementation complexity. For the applications targeted herein, a $10 \mathrm{Mbps}$ nominal data rate over a $1 \mathrm{GHz}$ transmit bandwidth is selected. Through the CCDM modulation, the data rates can be scaled from below $2 \mathrm{Mbps}$ to over $40 \mathrm{Mbps}$. The carrier frequency will be adjustable depending on the allocated frequency band, for example 6.5 GHz.

Due to the $1 \mathrm{GHz}$ transmit bandwidth, the impulse duration will be around 1 to $2 \mathrm{~ns}$. The chip time is set to $6.25 \mathrm{~ns}$, so that the impulse duty cycle is about $1 / 6$ to $1 / 3$, which will be suitable for concurrent transmission and reception.

Two different MCIDS spreading configurations are considered to allow the PHY protocol to support different implementation complexity requirements. As shown in Figure 2, the low complexity MCIDS scheme spreads a block of 16 symbols with a Walsh-Hadamard sequence set of length 16 (i.e., there are 16 sequences in the set). No cyclic prefix is added in this scheme. The spread spectrum signal (called a MCIDS block) lasts $1600 \mathrm{~ns}$ (256 samples) per symbol block. The maximum channel delay with which this system can cope is $100 \mathrm{~ns}$. The CCDM modulation before the MCIDS spreading will use a complementary code set of length 8. A more complicated MCIDS scheme uses a symbol block of size 60 , which is spread by a Kasami sequence set (small set) of length 15 (i.e., although there are only 4 sequences in the set, each sequence can be circularly shifted for a total of 60 sequences). A cyclic prefix is added in this case. The duration of the MCIDS block is $6000 \mathrm{~ns}$ (960 samples). A maximum channel delay of $375 \mathrm{~ns}$ can be coped with by this system. The CCDM modulation before the MCIDS spreading will also use a complementary code set of length 8 .

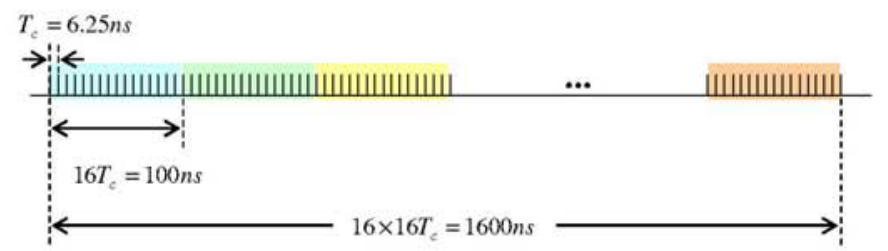

(a)

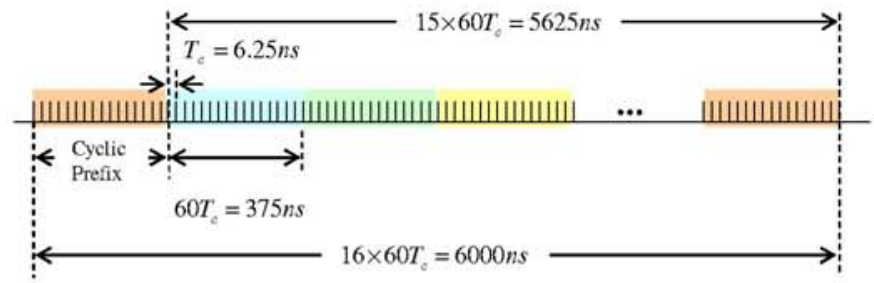

(b)

Figure 2. MCIDS spreading configurations: (a) 16 symbol block with WalshHadamard sequence set of length 16 and (b) 60 symbol block with Kasami sequence set of length 15 .

The PHY packet format will comprise a preamble of 8 or 2 MCIDS blocks, a combined PHY and MAC header of 40 or 10 MCIDS blocks, and a variable number of payload MCIDS 
blocks. All transmitted data symbols will be scrambled and protected by appropriate CRC parity checks.

Pilot symbols will be inserted in each data MCIDS block to facilitate channel estimation in the receiver. For the 16 symbol MCIDS block using Walsh-Hadamard sequence set, there are 12 data symbols and 4 pilot symbols, so that the effective data rate will be reduced to $7.5 \mathrm{Mbps}$. For the 60 symbol MCIDS block using Kasami sequence set, there are 48 data symbols and 12 pilot symbols, so that the effective data rate will be reduced to $8 \mathrm{Mbps}$.

\section{PHYSICAL LAYER PROTOCOL}

The PHY protocol described here assumes the low complexity MCIDS spreading configuration as shown in Figure 2 (a). The PHY frame contains three fields: preamble with start frame delimiter (SFD), header (including PHY header and MAC header) with header check sequence (HCS), and frame payload with frame check sequence (FCS), as shown in Figure 3. Stuff bits (SB) may be added following FSC in order to group payload and FCS bits into CCDM and MCIDS blocks.

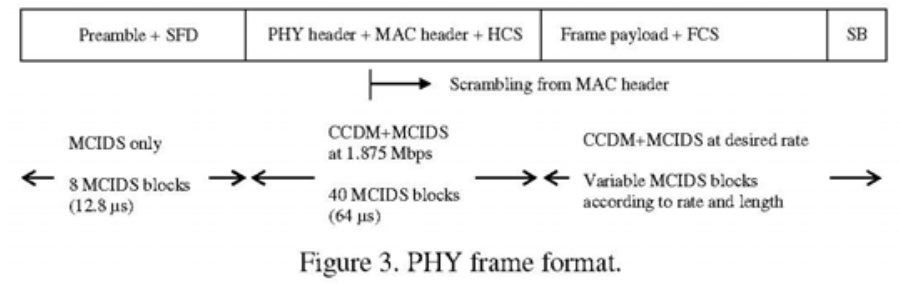

The preamble with SFD field consists of 8 MCIDS blocks and lasts $12.8 \mu \mathrm{s}$. Only the MCIDS spreading is applied in this field (i.e., no CCDM modulation). The header field consists of 40 MCIDS blocks under normal operation. The frame payload with FCS field contains variable MCIDS blocks according to the length of the payload and the date rate defined in the PHY header. The CCDM modulation starts from the PHY header. Data scrambling starts from the MAC header. Under concurrent $\mathrm{Tx} / \mathrm{Rx}$ operation mode, an outer code must be applied to the MICDS blocks starting from the header field.

There are three preamble options, one for normal operation (i.e., without concurrent $\mathrm{Tx} / \mathrm{Rx}$ ), two for concurrent $\mathrm{Tx} / \mathrm{Rx}$ operation with respective outer codes $\{+1,+1\}$ and $\{+1,-1\}$. The preamble is used primarily for the receiver to estimate the channel coefficients and achieve clock synchronization. The preamble consists of 6 MCIDS blocks. Each MCIDS block is generated by spreading 16 binary bits using the MCIDS spreading. Thus, there are 96 binary bits in the preamble.

There are also three SFD options in conjunction with the above defined preamble options. An SFD consists of a 4-bit pattern, which is spread by an 8-bit code before the MCIDS spreading. The resulting 32 binary bits will produce two MCIDS blocks. The 4-bit SFD pattern will be searched by the receiver to determine the frame timing and the outer code used in the incoming frame.

The PHY header consists of three octets that specify the seed identifier for the data scrambler, the data rate of the frame payload, and the number of octets in the frame payload (which does not include FSC or SB). The number of the MCIDS blocks produced by the frame payload (with FSC and SB) can be determined by the data rate and the payload length information.

The nominal symbol rate before MCIDS spreading is 10 Mbps. However, since 4 out of the 16 total symbols in a MCIDS block are used for pilots, the nominal data rate after the MCIDS spreading is reduced to $7.5 \mathrm{Mbps}$.

The PHY protocol defines six data rates: $1.875 \mathrm{Mbps}, 3.75$ Mbps, 7.5 Mbps, $15 \mathrm{Mbps}, 30 \mathrm{Mbps}$, and $45 \mathrm{Mbps}$. Each data rate is achieved by choosing a unique symbol mapping and symbol rate combination used in the CCDM modulation. Note that the CCDM nominal symbol rate is defined in the absence of an outer code (i.e. the concurrent transmit and receive mode is not operating). The CCDM symbol rate will be doubled when an outer code is used. Also note that there is no need for an explicit bit in the PHY header since a determination as to the presence of the outer code can be derived from the preamble.

The MAC header consists of 10 octets, which is the same as that defined in IEEE 803.15.3 standard [4].

The header field is transmitted using the lowest date rate of 1.875 Mbps.

The CCDM modulation uses a set of 8 orthogonal complementary code spreading sequences of length 64 . This set of orthogonal complementary code spreading sequences is used to modulate 8 data symbols. The size of one CCDM block is 96. Before the CCDM modulation, the binary serial input data bits need to be divided into groups of $1,2,4$, or 6 bits and converted into data symbols (complex numbers representing BPSK, QPSK, 16-QAM, or 64-QAM constellation points). The conversion is performed according to Gray-coded constellation mappings. The average power within a CCDM block is required to be a constant, regardless of the modulation. Thus, after the CCDM modulation, the output CCDM signal must be multiplied by a normalization factor.

The MCIDS spreading uses the Walsh-Hadamard sequence set of length 16. One MCIDS block will spread 12 CCDM signal samples and 4 pilot bits. The pilot bits are defined as $\{+1,-1,+1,-1\}$ and located in positions indexed $0,4,8$, and 12 in a 16-sample data block. Since a CCDM block has 96 signal samples, 8 MCIDS blocks are required to spread one CCDM block.

The PHY has two operation modes. One is the normal operation mode; the other is the concurrent $\mathrm{Tx} / \mathrm{Rx}$ operation mode. Under normal operation mode, the preamble pattern and SFD use option 1. No outer code is used for the MCIDS blocks in the header and payload fields of the PHY frame. Under concurrent $\mathrm{Tx} / \mathrm{Rx}$ operation mode, the preamble pattern and SFD use option 2 to indicate the use of the outer code $\{+1,+1\}$ in the header and payload fields, or, option 3 to indicate the use of the outer code $\{+1,-1\}$. Different outer codes must be used to differentiate transmission and reception paths.

When the transceiver starts to transmit while receiving a packet, the transmitter clock shall be delayed by half chip time (3.125 ns). The MCIDS block must be delayed by $1 / 4 \mathrm{~L}$ chip 
time, where $\mathrm{L}=16$ denoting the allowed maximum channel delay (in chip time).

The baseband signal bandwidth is defined as $1 \mathrm{GHz}$ at -10 $\mathrm{dB}$ cut off point. The transmitted signal power spectral density (PSD) shall be less than the limits shown in Figure 4 (solid line). Also shown in this figure are a practical transmit PSD (dotted-line), obtained using a rectangular pulse of width $1.5625 \mathrm{~ns}$ (1/4 chip time) filtered by a second-order Butterworth filter with $-3 \mathrm{~dB}$ cut-off frequency at $0.64 \mathrm{GHz}$, and the original rectangular pulse's PSD (dashed-line). After matched-filtering, the received signal pulse is shown in Figure 5 .

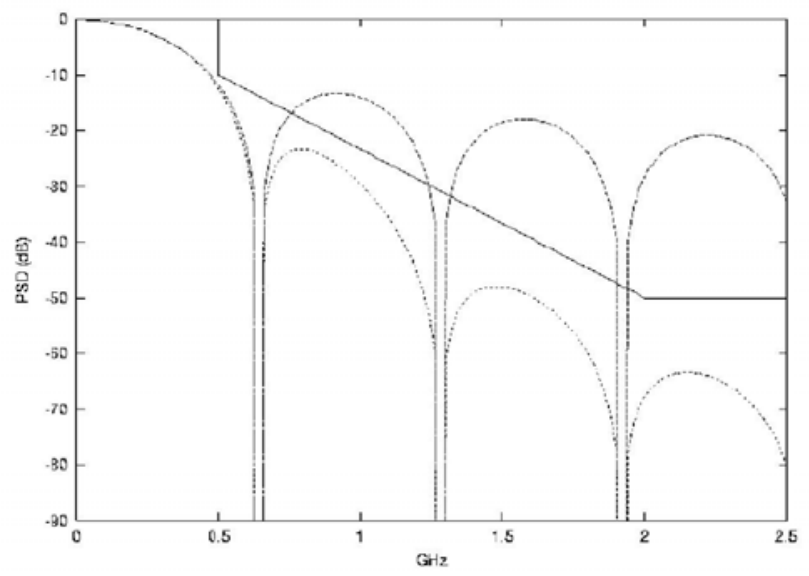

Figure 4. Transmit mask and a practical transmitted signal PSD.

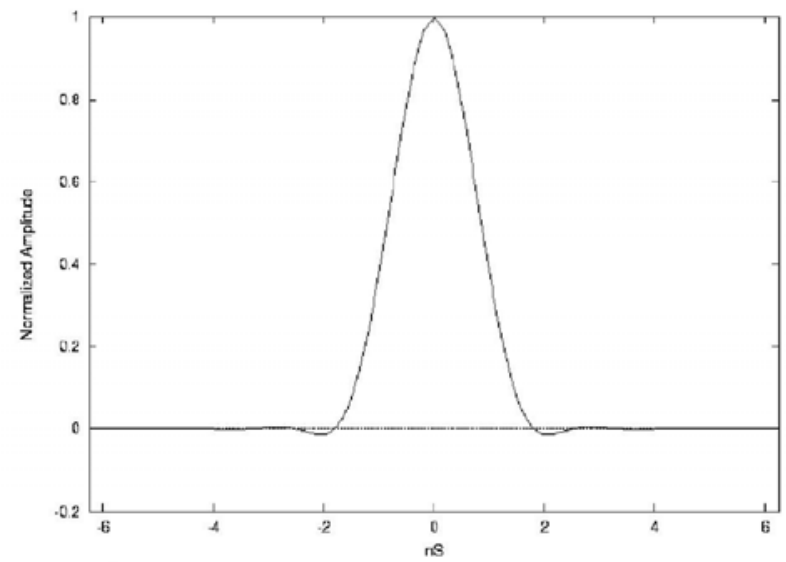

Figure 5. Received UWB baseband pulse (after matched filtering).

\section{PERFORMANCE SIMULATION}

The performance of the proposed UWB system is investigated using a developed simulation platform and practical receiver algorithms (i.e., taking into consideration of the channel estimation, signal synchronization and tracking) under different data rates and channel environments, which is measured in terms of bit-error-rate (BER). Figure 6 shows the block diagram of the system model used for the simulation, which consists of a transmitter, a wireless channel (with AWGN, multipath, and local and remote transmitter interferences), and a receiver. In addition, a random binary signal generator is included to provide the data bytes in the PHY frames composed by the transmitter. A BER analyzer is used to calculate the BER after the receiver detects the transmitted frames. Some simulation parameters are also listed below.

- Transmitter frame length: 1024 octets

- Transmitter data rate: varying according to modulations

- Transmitter chip time: $6.25 \mathrm{~ns}$

- Transmitter filter sampling rate: 16 samples/chip

- Receiver sampling rate (after matched filtering): 4 sample/chip

- Number of taps in multipath channel model: 3 64 taps

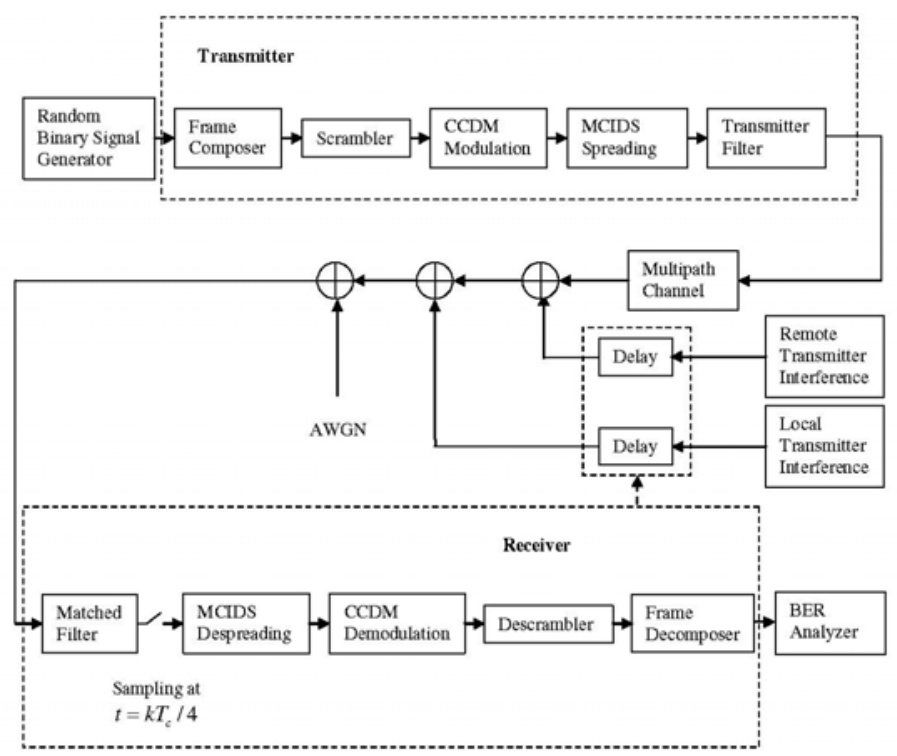

Figure 6. System model for UWB PHY simulation.

\section{A. Performance in Gaussian channel}

The performance of the proposed UWB system in Gaussian channel is shown in Figure 7, which is represented by the curves in terms of BER versus normalized signal-to-noise ratio $\left(\mathrm{E}_{\mathrm{b}} / \mathrm{N}_{0}\right)$. The results prove that the receiver algorithms are feasible in practice and the simulation platform is working. Note that, in this simulation, a 32 tap channel length is used. This means that the receiver actually assumes a multipath channel rather than a Gaussian channel. Compared with theoretical performance in Gaussian channel, there is a slight degradation for each data rate. This is because the synchronization is not perfect under noisy conditions and the channel estimation is also not perfect. In addition, there is a slight degradation for lower rate BPSK, as compared with QPSK. This indicates that the CCDM does not produce $100 \%$ coding gain without perfect channel estimation.

\section{B. Performance under concurrent Tx/Rx mode}

The performance under concurrent transmission and reception operation mode is also simulated in Gaussian channel. We assume that the local transmitter interference is $30 \mathrm{~dB}$ higher than the received signal and the remote transmitter interference is $0 \mathrm{~dB}$ relative to the received signal. We also use 
a 3-tap channel length in the simulation. This means that the receiver is dedicated to operate in Gaussian channel. The results are shown in Figure 8. We see that the performance in terms of bit-error-rate versus normalized signal-to-noise ratio is similar to that in normal operation mode. Note that under the same modulation scheme the actual data rate in concurrent transmission and reception mode will be only half of the data rate in normal operation mode due to the use of outer code. However, the signal power in concurrent transmission and reception mode is also only half of the signal power in normal operation mode under the same normalized signal-to-noise ratio. Therefore, with the same signal power, the actual data rate in concurrent transmission and reception mode can be increased by using higher rate modulation scheme.

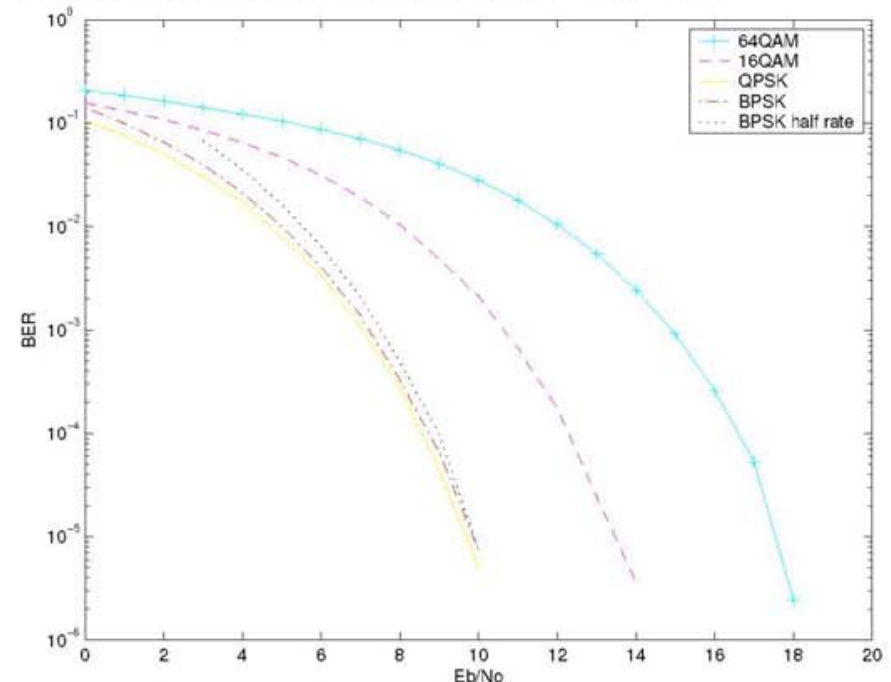

Figure 7. Performance of proposed UWB receiver algorithms in Gaussian channel.

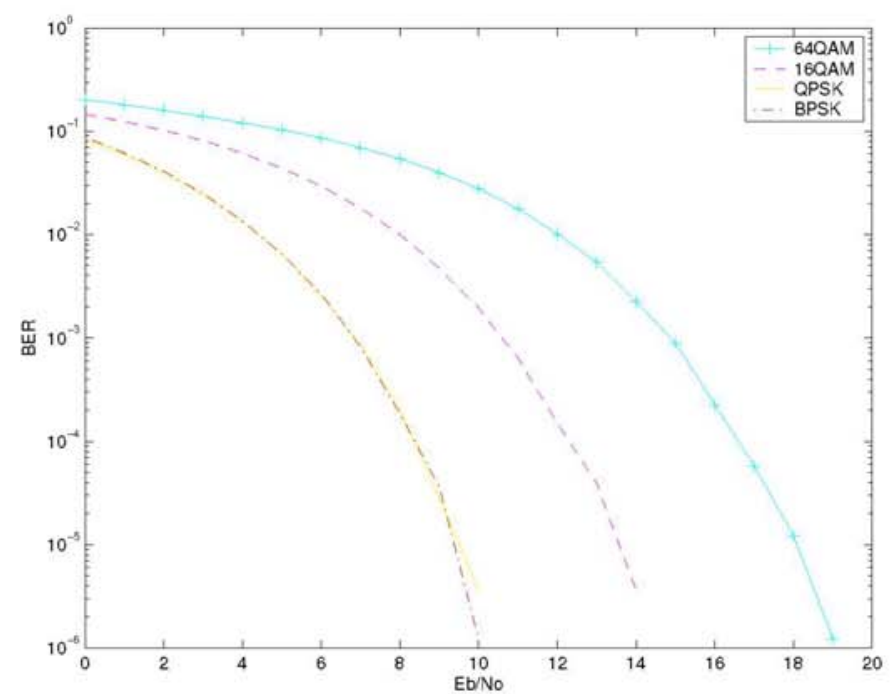

Figure 8. Performance of proposed UWB receiver algorithms in Gaussian channel under concurrent Tx/Rx mode.

\section{Performance in multipath channel}

The performance of the proposed UWB system using practical receiver algorithms under multipath channel is simulated for the IEEE channel model CM1 [5]. The results are shown in Figure 9. The receiver uses a 64-tap channel length, which means that $100 \mathrm{~ns}$ maximum path delay can be coped with. For the CM1 channel model, most path delays are within $50 \mathrm{~ns}$, so that the performance is only slightly degraded compared with Gaussian channel performance.

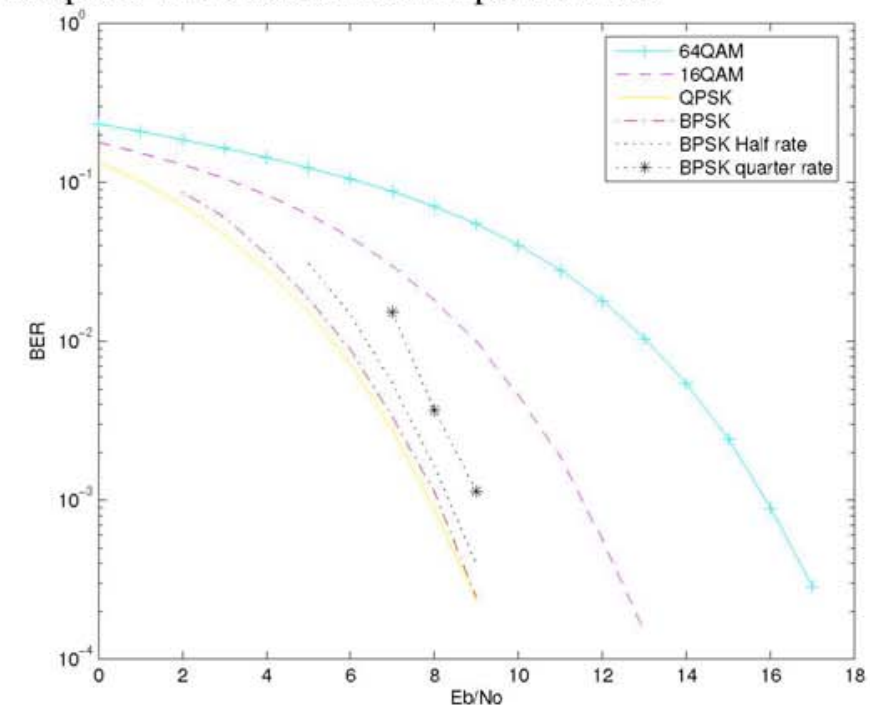

Figure 9. Performance of proposed UWB receiver algorithms in multipath channel CM1.

\section{CONCLUSIONS}

To demonstrate the performance of the new UWB system proposed for low power, long range, mid to high data rate mesh networking applications, the transceiver architecture and PHY protocol are designed, and the simulation platform and practical receiver algorithms for performance evaluation are developed. The simulation results prove that the designed transceiver architecture and PHY protocol are adequate and that the developed algorithms are feasible for practical implementation. Future work includes improving the SFD detection performance and carrying out more simulation to evaluate system performance in terms of frame-loss-rate and frame-error-rate. The algorithms for concurrent $\mathrm{Tx} / \mathrm{Rx}$ mode under multipath channel condition will also be developed. More results will be produced under other IEEE channel models. A radio propagation model will be incorporated in the simulation platform to determine the operation range.

\section{REFERENCES}

[1] X. Huang, D. Lowe, R. Gandia, and E. Dutkiewicz, "An impulse ultrawideband system capable of concurrent transmission and reception, part I: requirements and innovations," presented at The 2006 International Conference on Communications, Circuits and Systems (ICCCAS2006), Guilin, China, 25-28 June, 2006.

[2] R. Kohno et al., "DS-UWB physical layer submission to 802.15 task group 3a," IEEE P802.15-04/0137r1, May 2004.

[3] A. Batra et al., "Multi-band OFDM physical layer proposal for IEEE 802.15 task group 3a," IEEE P802.15-03/268rl, September 2003.

[4] IEEE Standard P802.15.3/D17, "Part 15.3: wireless medium access control (MAC) and physical layer (PHY) specifications for high rate wireless personal area networks (WPAN)," February 2003.

[5] J. Foerster (editor), "Channel modelling sub-committee report final," IEEE802.15-02/490, March 2003. 